\title{
Novel developmental bases for the evolution of hypobranchial muscles in vertebrates
}

\author{
Rie Kusakabe ${ }^{1 *} \mathbb{D}$, Shinnosuke Higuchi ${ }^{1,2,3}$, Masako Tanaka', Mitsutaka Kadota ${ }^{4}$, Osamu Nishimura ${ }^{4}$ and \\ Shigeru Kuratani ${ }^{1,5}$
}

\begin{abstract}
Background: Vertebrates are characterized by possession of hypobranchial muscles (HBMs). Cyclostomes, or modern jawless vertebrates, possess a rudimentary and superficial HBM lateral to the pharynx, whereas the HBM in jawed vertebrates is internalized and anteroposteriorly specified. Precursor cells of the HBM, marked by expression of $L b \times 1$, originate from somites and undergo extensive migration before becoming innervated by the hypoglossal nerve. How the complex form of HBM arose in evolution is relevant to the establishment of the vertebrate body plan, but despite having long been assumed to be similar to that of limb muscles, modification of developmental mechanisms of HBM remains enigmatic.
\end{abstract}

Results: Here we characterize the expression of Lbx genes in lamprey and hagfish (cyclostomes) and catshark (gnathostome; jawed vertebrates). We show that the expression patterns of the single cyclostome Lbx homologue, $L b x-A$, do not resemble the somitic expression of mammalian $L b x 1$. Disruption of $L b x-A$ revealed that $L j L b x-A$ is required for the formation of both HBM and body wall muscles, likely due to the insufficient extension of precursor cells rather than to hindered muscle differentiation. Both homologues of $L b x$ in the catshark were expressed in the somitic muscle primordia, unlike in amniotes. During catshark embryogenesis, $L b \times 2$ is expressed in the caudal HBM as well as in the abdominal rectus muscle, similar to lamprey $L b x-A$, whereas $L b x 1$ marks the rostral HBM and pectoral fin muscle.

Conclusions: We conclude that the vertebrate HBM primarily emerged as a specialized somatic muscle to cover the pharynx, and the anterior internalized HBM of the gnathostomes is likely a novelty added rostral to the cyclostome-like HBM, for which duplication and functionalization of $L b x$ genes would have been a prerequisite.

Keywords: Hypobranchial muscle, Development, Lamprey, Hagfish, Shark, Skeletal muscle, Lbx genes, Evolution, Vertebrates

\footnotetext{
* Correspondence: rie.kusakabe@riken.jp

'Laboratory for Evolutionary Morphology, RIKEN Center for Biosystems

Dynamics Research (BDR), 2-2-3 Minatojima-minami, Chuo-ku, Kobe, Hyogo

650-0047, Japan

Full list of author information is available at the end of the article
}

(c) The Author(s). 2020 Open Access This article is licensed under a Creative Commons Attribution 4.0 International License, which permits use, sharing, adaptation, distribution and reproduction in any medium or format, as long as you give appropriate credit to the original author(s) and the source, provide a link to the Creative Commons licence, and indicate if changes were made. The images or other third party material in this article are included in the article's Creative Commons licence, unless indicated otherwise in a credit line to the material. If material is not included in the article's Creative Commons licence and your intended use is not permitted by statutory regulation or exceeds the permitted use, you will need to obtain permission directly from the copyright holder. To view a copy of this licence, visit http://creativecommons.org/licenses/by/4.0/ The Creative Commons Public Domain Dedication waiver (http://creativecommons.org/publicdomain/zero/1.0/) applies to the data made available in this article, unless otherwise stated in a credit line to the data. 


\section{Background}

Many characteristics of vertebrate body plan are associated with the extremely complex morphology of skeletal muscles, as compared to the simple contractile tissues of invertebrate chordates, such as those found in amphioxus. Of the vertebrate skeletal muscles, hypobranchial muscles (HBMs), including tongue and infrahyoid muscles in jawed vertebrates, have attracted particular attention in evolutionary studies, due to their peculiar developmental processes. Precursor cells of the HBM originate from somites and migrate along an extensive, arch-shaped pathway circumventing the posterior margin of the posteriormost pharyngeal arch, as a cell stream called the hypoglossal cord [1]. HBMs become innervated by the hypoglossal nerve, the twelfth cranial nerve that runs through the so-called head/trunk interface, overlapping with the hypoglossal cord [2].

The extensive migration of the somitic muscle precursors has been best studied in the formation of paired limbs, another feature shared by vertebrates. At the foreand hindlimb levels, the ventrolateral lip (VLL) of the epithelial dermomyotome yields de-epithelialized, mesenchymal precursor cells for the limb muscles. The limb muscle precursors lose their original segmental configuration, forming a pool of mesenchymal progenitor cells that migrate distally into the limb buds, where they undergo muscle differentiation [3]. These precursor cells are marked by the expression of $L b x 1$, a member of lady bird class of homeobox transcription factor-encoding genes [4, 5]. In $L b x 1$-deficient mice, the limb muscles are lost or severely affected, with tongue muscles reduced in size $[5,6]$. However, $L b x 2$, a paralogue of $L b x 1$, has never been proposed to be involved in myogenesis in either tongue or limbs [7-9].

In recent developmental studies, the cyclostome lampreys have served as an attractive model that retains the ancestral features of vertebrates [10-14]. One such characteristic is found in the simple morphology of skeletal muscles, e.g., a lack of epaxial/hypaxial distinction of the trunk muscles, as well as the paired fins and associated muscles $[15,16]$. On the other hand, lampreys, while lacking the tongue, do possess a putative HBM homologue lying in the superficial layer of the pharyngeal wall, innervated by a nerve called the hypoglossal nerve (XII) in the lamprey (Fig. 1a) [18, 19]. Precursors of the lamprey HBM appear to originate from the ventral side of rostral somites of the late pharyngula (stage 27) [20], first migrating ventrally within the pericardium and then extending anteriorly along the lateral aspect of the pharynx [11, 18, 21-23]. Muscle differentiation of HBMs takes place at the pre-ammocoete larval stage (stage 29; Additional file 1: Fig. S1j), much later than that of other somite-derived muscles. Upon differentiation, HBM myofibers secondarily acquire segmental pattern in conjunction with the pharyngeal arch muscles that derive from unsegmented head mesoderm (Additional file 1: Fig. S1b-f), but not in conjunction with segmentation of the dorsal somitic muscles dorsally overlying the pharyngeal arches (Additional file 1: Fig. S1k and l) [10, 22].

The lampreys, as well as the hagfish, the other group making up the cyclostomes, possess a single cyclostomespecific homologue of the $L b x$ gene, $L b x-A$ (Fig. S2). In the Japanese lamprey Lethenteron camtschaticum (formerly Lethenteron japonicum), Lbx-A ( $j j L b x-A)$ mRNA has been shown to mark the anteriorly extending HBM precursors [10]. This observation led us to speculate that $L j L b x-A$ would retain the ancestral myogenic function of $L b x$ gene in HBM formation acquired early in vertebrate evolution. However, it remains unclear how $L b x 1 / L b x 2$ genes of gnathostomes are functionally related to the single $L b x$ gene of the lamprey. In this study, we aimed to clarify how the duplicated $L b x$ genes are involved in the morphological complexity of gnathostome HBM by comparing myogenesis in the lamprey, hagfish, and shark.

\section{Results and discussion \\ Characterization and functional analysis of the cyclostome $L b x-A$ genes}

In the present study, we first examined the functional importance of lamprey $L b x-A$ gene during development. Since $L j L b x-A$ sequence reported previously had lacked the $5^{\prime}$ part of the coding region [10], we isolated a fulllength cDNA clone of $L j L b x-A$ utilizing the updated genomic data and the gene modeling (Additional file 1: Fig. S2). Newly identified N-terminus of deduced amino acid sequence of $L j L b x-A$ contained a 6-amino acid lady bird domain which is shared with Drosophila lady bird and mammalian $L b \times 2$ genes, but not with mammalian $L b x 1$ genes, implicating a possible functional correlation of $L j L b x-A$ to $L b x 2$ genes of jawed vertebrates [24].

We subsequently carried out the detailed analyses of the expression of $L j L b x-A$. In addition to the expression in HBM (Fig. 1b-d), $L j L b x-A$ was also expressed in the ventral edge of the postotic trunk somites across the anteroposterior axis, in addition to the HBM precursor cells (Fig. 1e). These $L j L b x$-A-expressing cells in the trunk extend ventrally to form the body wall muscle (stage 29; Additional file 1: Fig. S1j), differentiating much later than the dorsal part of the trunk muscle (Fig. 1f, Additional file 1: Fig. S1g-j). In the later ammcoete larval stage ( $\sim 50 \mathrm{~mm}$ body length), $L j L b x-A$ was expressed in a cell layer at the dorsal edge of the myotomes, which would later give rise to the muscles in the dorsal median fin (Additional file 1: Fig. S4) [25].

The broad expression of $L j L b x-A$ in the ventral side of the trunk does not resemble the somitic expression of 


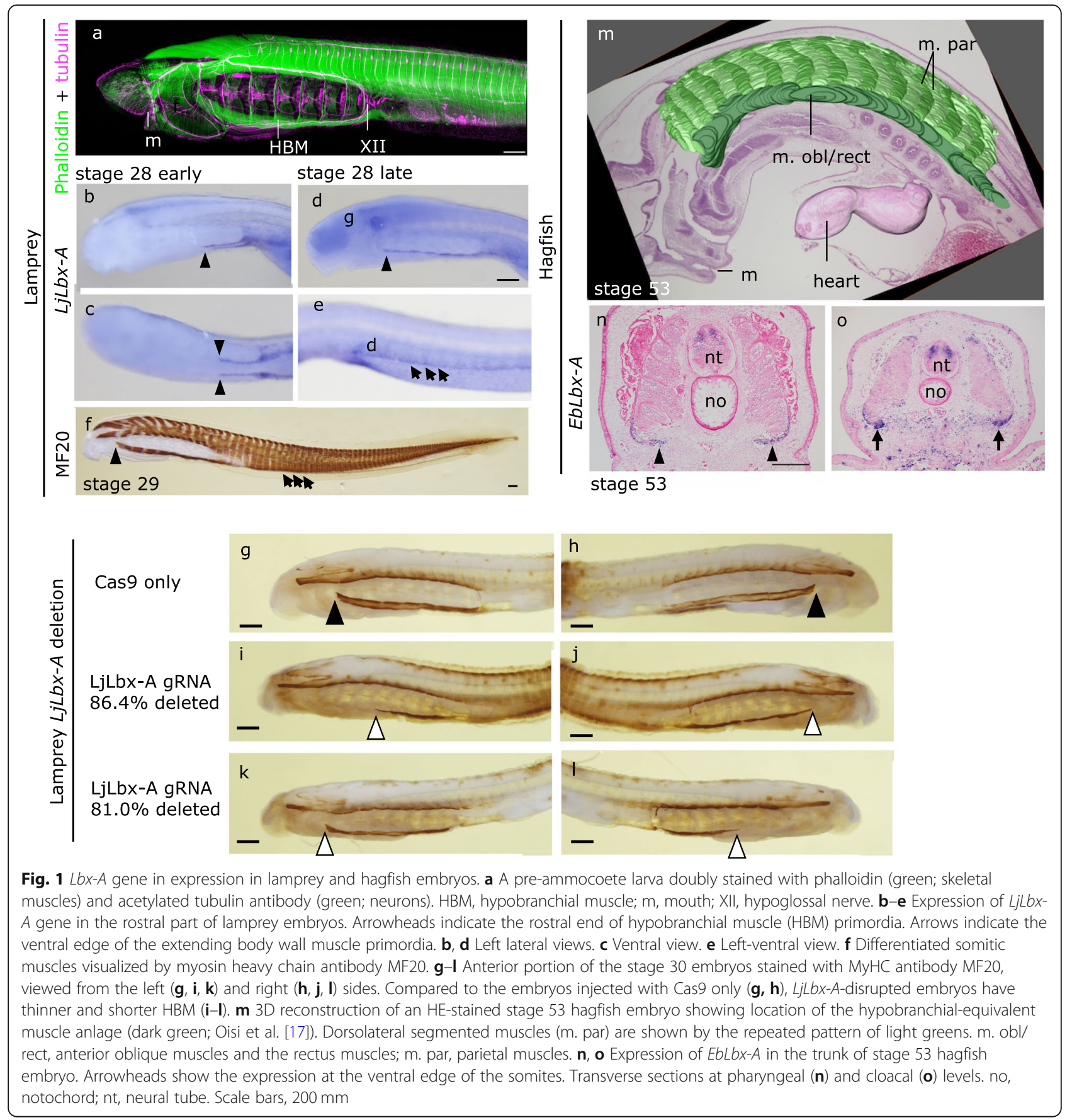

mammalian $L b x 1$, which is expressed only in the occipital and limb levels, but not in the flank $[4,26]$. To clarify which of the lamprey larval muscles require the function of $L j L b x-A$, we disrupted the $L j L b x-A$ locus during embryogenesis, using CRISPR/Cas9-mediated gene editing (Fig. 1g-l, Additional file 1: Fig. S5 to S8). After injection of the Cas9 protein and gRNA complementary to $L j L b x$ $A$, the dorsal somitic muscles of the larvae appeared unaffected, whereas HBM was lost partly or entirely from the ventral floor of the pharynx (Fig. 1g-l, Additional file
1: Fig. S6a-c). These embryos also lost body wall muscles, suggesting that $L j L b x-A$ is required for the formation of both HBM and body wall muscles (Additional file 1: Fig. S6d-f). We also found that $L j L b x$ - $A$-depleted embryos lost the expression of $L j P a x 3 / 7-A$ in the pharyngeal region, which marks the extending HBM primordium (Additional file 1: Fig. S7) [10, 23], suggesting that the loss of HBM and body wall muscles is attributable to the insufficient extension of precursor cells rather than to hindered muscle differentiation. 
We also characterized the $L b x$ gene of the hagfish Eptatretus burgeri, EbLbx-A (Additional file 1: Fig. S3). In hagfish embryos, $E b L b x-A$ was expressed in the ventral edge of the somites (Fig. 1n, o) [17]. This region has been reported to give rise to the anterior oblique muscles (m. decussatus) and the rectus muscles in the ventrolateral aspect of the pharyngeal wall $(\mathrm{m} . \mathrm{obl} / \mathrm{rect}$; Fig. $1 \mathrm{~m}$ ) [17]. M. obl/rect have been proposed to characterize the HBM of the hagfish, despite the fact that these muscles are innervated by the occipitospinal nerves that pass ventrally as individual segmental nerves, not by a bundled hypoglossal nerve which is the case in the lamprey HBM (Fig. 1a). The morphology of hagfish $\mathrm{m}$. obl/rect also differs significantly from that of the lamprey HBM; the oblique muscles broadly cover the surface of the ventral body from caudal to the mouth to the cloaca, and the rectus muscles lie longitudinally close to the ventral midline. Nevertheless, $\mathrm{m}$. obl/rect have been reported to differentiate at a later stage of embryogenesis [17], which is also the case in the development of the lamprey HBM and body wall muscles (Additional file 1: Fig. S1). The fact that the expression of $E b L b x-A$ is restricted to the ventral somites supports the HBM nature of the $\mathrm{m}$. obl/rect, suggesting that the $L b x$-dependent regulation of differentiation would be conserved in the cyclostomes.

\section{Catshark $L b \times 1$ and $L b \times 2$ are differentially expressed during myogenesis}

We chose the elasmobranch cloudy catshark Scyliorhinus torazame as a model to investigate how the wide variety of somite-derived hypaxial muscles, such as those in the limbs, appeared in the vertebrate species diverged early in evolution. It has been reported that, similarly to those of Osteichthyan animals, the $L b x 1$ gene of shark and skates are expressed in precursor cells in muscles [27, 28]. However, involvement of $L b x 2$ in myogenesis in these animals had remained unexplored, and no insights had been obtained with respect to the differential expression of $L b x 1$ and $L b x 2$, which emerged possibly due to the two rounds of vertebrate whole genome duplication (Additional file 1: Fig. S3) [27, 29]. In this study, we examined the expression of $L b x 1$ and $L b x 2$ during embryogenesis of $S$. torazame, using probes specific to each of the two genes. Unlike in mammals, both $L b x 1$ and $L b \times 2$ were expressed in the somitic muscle primordia, although in a non-overlapping fashion, as detailed below (Fig. 2).

Expression of catshark $L b x 1$ commenced at the epithelial VLL of postotic somites and became restricted to the pectoral and pelvic fin levels (Fig. 2a-c). Near the fin buds, these $L b \times 1$-positive cells detached from the VLL in bulk, in a similar configuration to the "muscle bud" described by Goodrich (Fig. 3a) [30, 31], to invade the fin bud, where they differentiated into adductor and abductor muscles of the paired fins (Figs. $2 a-d$ and $3 d, g$, j). Remarkably, these cell aggregates maintained their initial somitomeric pattern and were positive for ZO-1 (zona occuludens-1) antibody, a marker for tight junctions, suggesting that the dermomyotome persisting in the fin muscle primordia is epithelial in nature (Fig. 3e) [32, 33]. This observation is consistent with the classical view that the chondrichthyan fin muscles form from epithelial muscle primordium [30]. $L b x 1$ was also expressed in the muscle primordia in median fins (Figs. $2 \mathrm{~d}$ and $3 \mathrm{k}, \mathrm{l}$ ), similarly to the case in $L j L b x-A$ (Additional file 1: Fig. S4).

Catshark $L b \times 1$ was not expressed in the abdominal muscle primordia (Fig. 3a, g), unlike lamprey/hagfish $L b x-A$. Remarkably, in contrast to the $L b x 1$, catshark $L b x 2$ continued to be expressed in the VLL throughout the trunk (Figs. 2e-g and 3b). Lbx2-positive cells did not enter the fin buds; Lbx2-positive VLL cells extended ventromedially, passing through the medial aspect of the fin buds (Fig. 3b, f), marking the future abdominal rectus muscle (Fig. 3i). Thus, catshark $L b \times 2$, but not $L b \times 1$, marks the developing abdominal muscle, a feature similar to that of lamprey $L b x-A$ described above (Fig. 1e).

\section{Catshark HBM exhibits differential expression of $L b \times 1$ and Lbx2 during HBM development}

Our observations also suggested the differential functions of $L b \times 1$ and $L b \times 2$ in HBM development in S. torazame (Fig. 4). In early embryos, $L b x 2$ is expressed in a projection of the cells originating from the anterior somites (Fig. 4a, b, Additional file 1: Fig. S9a). The epithelial VLL of the 3rd and 4th somites released the Lbx2positive cells ventrally along the posterior edge of the pharynx (Additional file 1: Fig. S9a-c). Later, the Lbx2positive cells accumulated adjacent to the heart, then further proceeded anteriorly within the body wall ventral to the pharynx (Fig. 4a, b; Additional file 1: Fig. S9d-f). In contrast, the expression of $L b x 1$ was not consecutive along the posterior circumference of the pharynx. At stage 28, a single patch of $L b x 1$ expression became evident at the level of the 3rd pharyngeal arch (arrowhead in Figs. 2c and 4c, d, e'). This expression of $L b x 1$ in the anteriormost part of HBM primordium did not overlap with the $L b \times 2$ expression in the midline, whereas dorsally the leading edges of the bilateral portion of HBM transiently express both $L b x 1$ and $L b x 2$ (Fig. $4 \mathrm{f}^{\prime}$ and $\mathrm{f}^{\prime \prime}$ ). During the extension of HBM primordium from the somites, the anteriormost portion of $L b \times 2$-positive domain seems to provide $L b x 1$-positive precursor cells to give rise to the anterior HBM that fuses in the midline.

In late embryos, rows of $L b x 2$-positive cells have differentiated into coracoarcualis $(\mathrm{CAC})$ muscle, the posterior paired domain of HBM (Fig. 4g, g', h) [34]. CAC myofibers consist of 4 segments each of which 


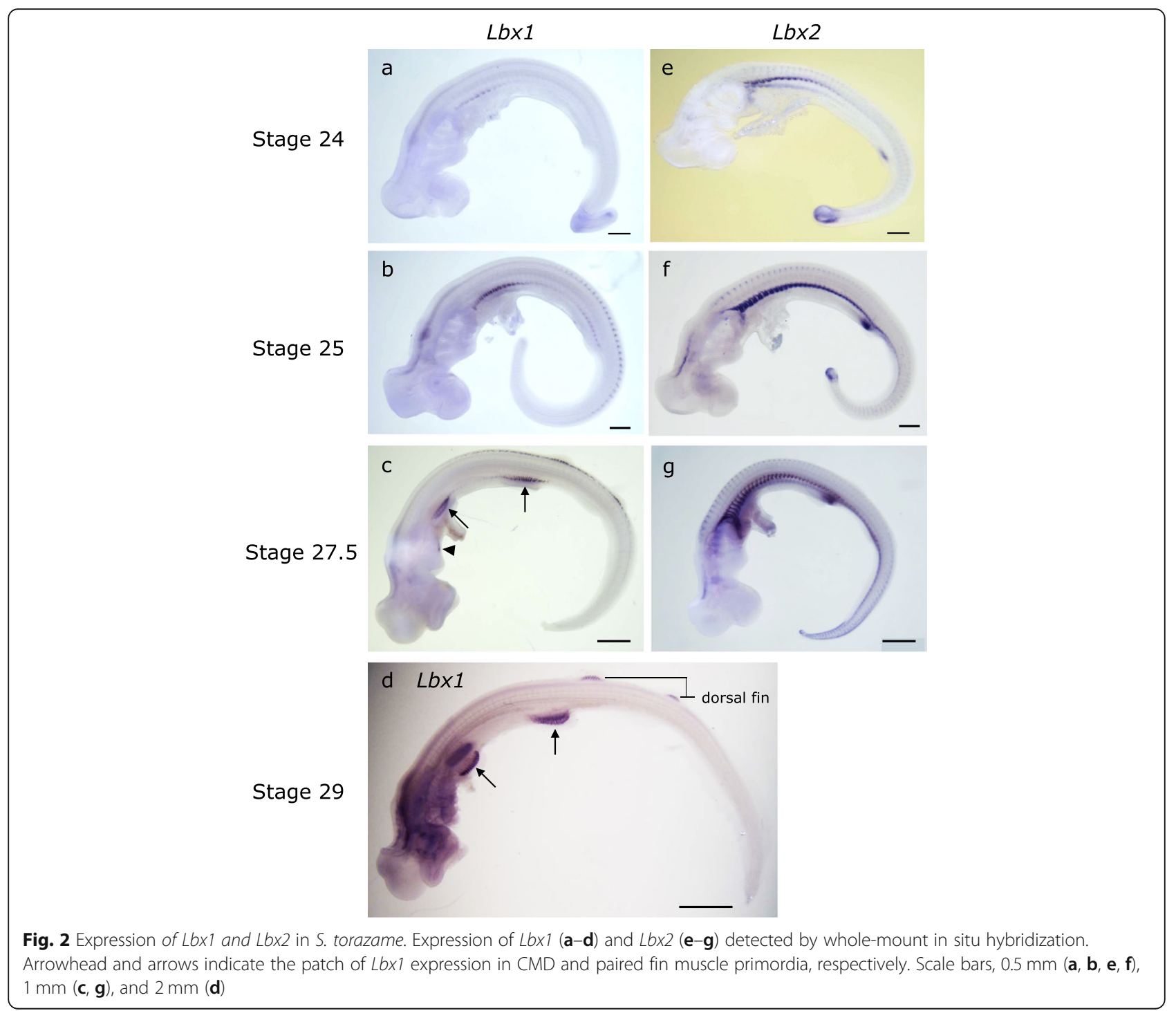

corresponding to the posterior pharyngeal arches (Fig. 4l, $\mathrm{m}$; pa3-6), a remarkable similarity to the lamprey HBM (Additional file 1: Fig. S1l). Anatomically, CAC originates at the scapulocoracoid cartilage and inserts to the anterior, medially located HBM (coracomandibularis muscle, CMD; Fig. 4i, j), a morphological orientation consistent with that of the amniote rectus cervicus (sternohyoideus) muscle [11, 34].

Lbx1-positive cells, on the other hand, differentiated later than $\mathrm{CAC}$, giving rise to CMD muscle in the midline, located anterior to the CAC muscle (Fig. 4i, j). Unlike the CAC muscle, the CMD muscle was composed of a long single segment of myofibers and inserted to the Meckel's cartilage (Fig. 4k, l), reminiscent of mammalian geniohyoideus muscle [34].

It is also noteworthy that the entire HBM primordia, including both $L b x 1$ - and $L b \times 2$-positive cell populations, were stained with the ZO-1 antibody (Additional file 1:
Fig. S10). This observation suggests that, in the shark, both the HBM precursor cells and the fin muscle primordia are epithelial in nature, and both remain as a coherent aggregate during the process of extension into the distal parts of the body.

\section{Duplicated gnathostome $L b x$ genes and complexity of skeletal muscles}

These results provide a new scheme for the developmental homology of HBM elements and appendicular muscles of the vertebrates (Fig. 5). The catshark CAC muscle (associated with $L b x 2$ expression) and the lamprey HBM (associated with $L b x-A$ expression) are both attached with the ventral ends of pharyngeal muscles and do not fuse in the midline (Fig. 5b, c) [11]. Moreover, catshark CAC and lamprey HBM are also similar with respect to the pattern of myofiber segmentation which corresponds to the adjacent pharyngeal arches 


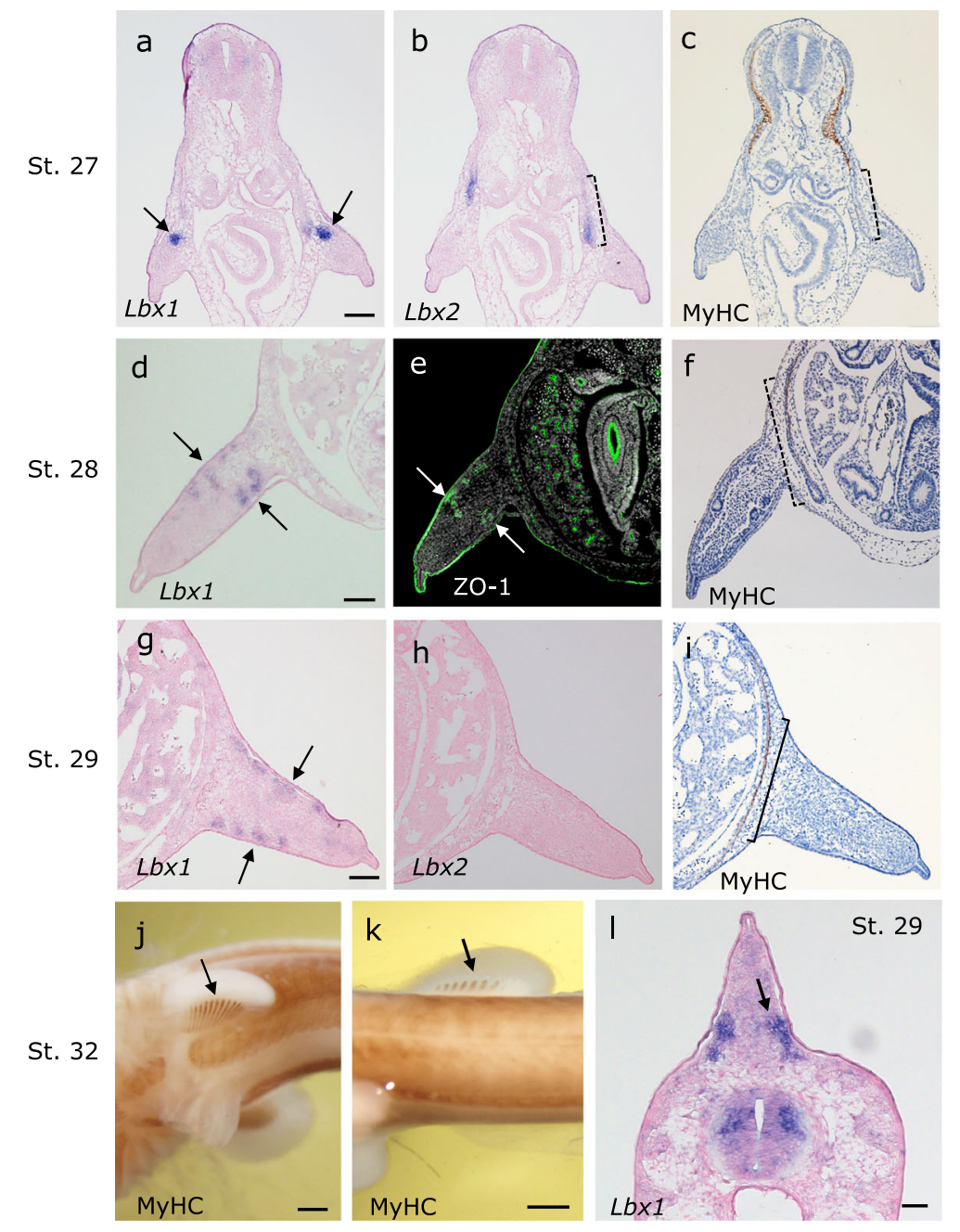

Fig. 3 Shark $L b \times 1$ and $L b \times 2$ are expressed in distinct muscle primordium in the trunk. Transverse sections of S. torazame embryos at a-c stage 27, d-f stage 28 , and $\mathbf{g}$-i stage 29. a Expression of $L b \times 1$ detected in the pectoral muscle precursor cells (arrows). $\mathbf{b}$ A serial section to $\mathbf{a}$, $L b \times 2$ is expressed in the ventral somite in the body wall. c At the pectoral fin level, $L b \times 2$ is expressed in rectus abdominus muscle primordia (dotted bracket), which is not yet differentiated, as shown by the absence of MyHC staining (c). $\mathbf{d}, \mathbf{e} L b \times 1$ is expressed in the pectoral fin muscle primordia (arrow) which is also stained with ZO-1 tight junction antibody. $\mathbf{g - i}$ At stage 29, Lbx1 is expressed in the pectral fin muscle primordia which are still not differentiated. Expression of $L b x 2$ is no longer detectable. Muscle differentiation is observed only in the rectus abdominus (dotted bracket) but not yet in the fin (i). $\mathbf{j}, \mathbf{k}$ At stage 32, muscle differentiation is observed in the appendicular (arrow in $\mathbf{j}$ ) and median (arrow in k) fins. I At stage 29, $L b x \mathbf{l}$ is expressed in the muscle precursor cells in the dorsal median fin (arrow). Scale bars, a, d, g 0.1 mm; j, k 0.5 mm; $10.05 \mathrm{~mm}$

(Additional file 1: Fig. S1k and l; Fig. 4m; Fig. 5b, c), suggesting their muscle differentiation is under the influence of pharyngeal embryonic components such as cephalic neural crest cells and the cranial mesoderm cells. On the other hand, Lbx1-positive CMD of the shark, which is homologous to the tetrapod geniohyoideus and genioglossus muscles, seems to be a novel muscular component acquired in gnathostomes (Fig. 5c). CMD-equivalent muscles, as well as the appendicular muscles, are lacking in the cyclostomes, whose HBM is entirely bilateral and segmented in accordance with the pharyngeal arches.
Considering expression patterns of $L b x 1 / L b x 2$ genes in the wide variety of Osteichthyans, however, the evolutionary pathway seems more complex. In amphibians, only the $L b x 1$ gene has been identified in genomic sequences, exhibiting a loss of $L b \times 2$ locus in the amphibian lineage (Additional file 1: Fig. S3) [8, 29]. In Xenopus laevis and direct-developing frog Eleutherodactylus co$q u i, L b x 1$ is expressed in the ventral side of the trunk somites [35, 36]. In Xenopus, these Lbx1-positive somitic cells give rise to the "rectus abdominus" muscle that extends from the ventral edge of the somites and eventually surrounds the abdomen of tadpoles, similarly to the 


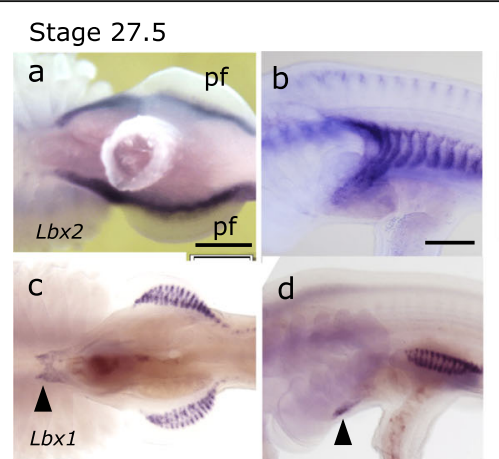

Stage 29
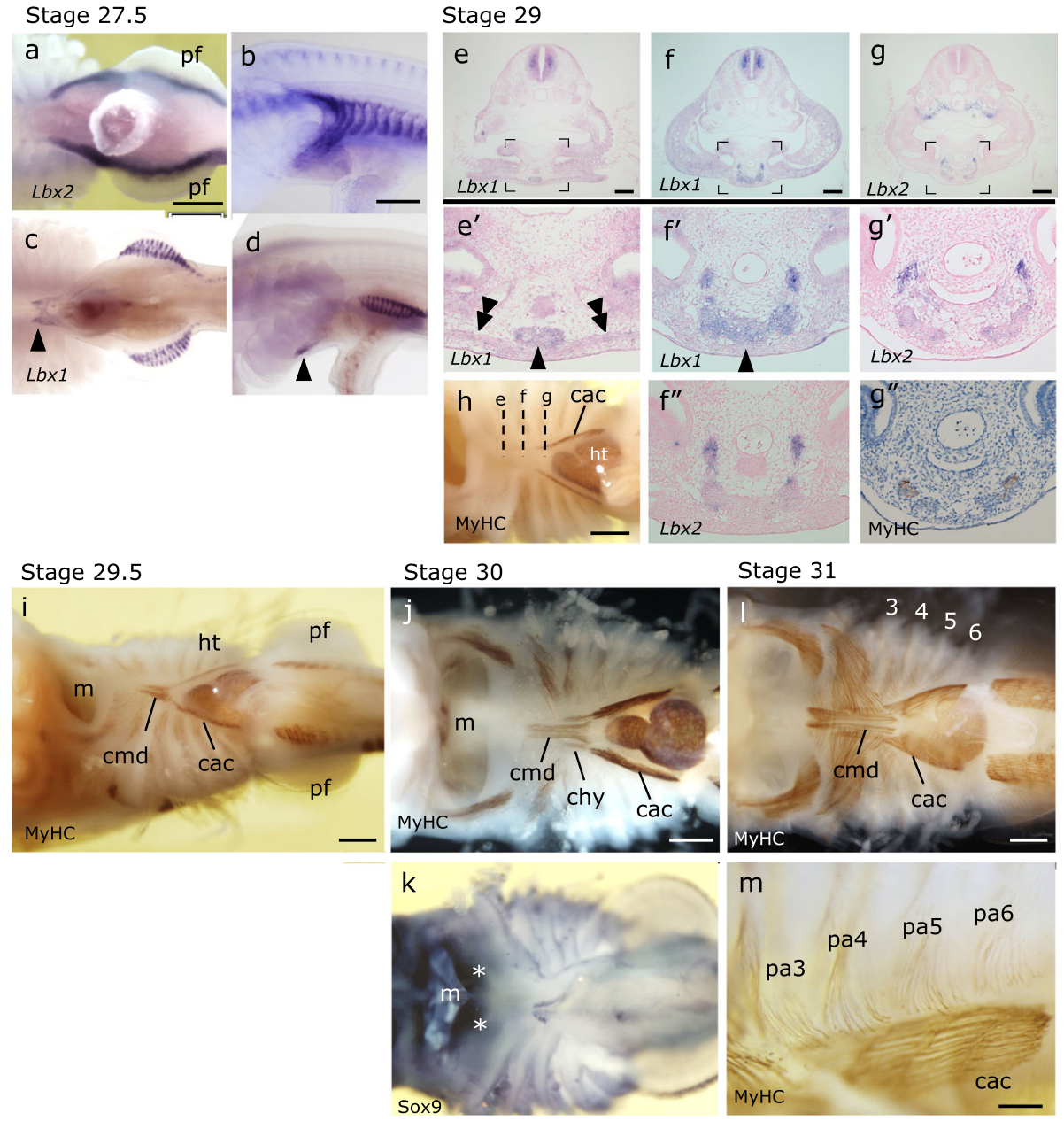

Fig. 4 Anterior part of the shark hypobranchial muscles is marked by the expression of $L b x 1$. a-d Whole-mount in situ hybridization of S. torazame embryos at stage 27.5. a, b Expression of $L b \times 2$ in ventral (a) and left lateral (b) views. c, d Expression of $L b \times 1$ in ventral (c) and left lateral (d) views. Arrows show the expression in the anterior extremity of HBM primordium. $\mathbf{e}-\mathbf{g}$ " Transverse sections of a stage 29 embryo at the levels indicated in $\mathbf{h}$ (ventral view stained with MyHC antibody). $\mathbf{e}^{\prime}-\mathbf{g}^{\prime}$ The enlarged views of the areas marked in $\mathbf{e}-\mathbf{g} . \mathbf{f}^{\prime \prime}$ and $\mathbf{g}^{\prime \prime}$ are the adjacent sections to $\mathbf{f}^{\prime}$ and $\mathbf{g}^{\prime}$, respectively, hybridized with different probes. $\mathbf{e}^{\prime} L b x 1$ is expressed in the medial HBM precursor cells (arrowhead) located dorsal to the hyoid archderived muscles (double arrowheads). $\mathbf{f}^{\prime}, \mathbf{f}^{\prime \prime} L b \times 1$ is expressed in the medial HBM precursor cells, whereas $L b \times 2$ expression is restricted in the bilateral HBM primordium $\left(\mathbf{f}^{\prime \prime}\right) . \mathbf{g}^{\prime}, \mathbf{g}^{\prime \prime}$ Earliest differentiation of HBM occurs in the cells in which $L b \times 2$ is downregulated. i At stage 29.5, the anterior medial part of HBM differentiates as coracomandibularis (cmd) muscle. $\mathbf{j}$ At stage 30 , the anterior tip of early $\mathrm{m}$. coracoarcualis (cac, originally Lbx2-positive) extended anteriorly, forming coracohyoideus (chy) muscle connecting cac muscle and basihyal cartilage, overlapping laterally with $\mathrm{m}$. coracomandibularis. Coracomandibularis now forms a single medial bundle of muscle fibers that connects the coracoarcualis muscle and the lower jaw. k Meckel's cartilage expressing Sox9 (asterisk). I, $\mathbf{m}$ The cac muscle has four segments corresponding to the adjacent pharyngeal arches (pa3-6). I Ventral view. $\mathbf{m}$ Left ventrolateral view. ht, heart; m, mouth. Scale bars, a-I 0.5 mm; $\mathbf{m} 0.2$ mm

body wall muscles of other vertebrates. Xenopus $L b x 1$ is also expressed in the limb muscle precursors that appear during the metamorphosis [35]. In zebrafish, both $L b x 1 b$ and $L b x 2$ genes were shown to be involved in hypaxial muscles including pectoral fin muscles $[8,37,38]$. It has been suggested that the primary role of $L b x$ transcription factors is to control the switch of proliferation/differentiation of the muscle precursor cells $[8,39]$. $L b x 1$ and $L b \times 2$ have been suggested to possess overlapping regulatory functions, as the forced expression of $L b x 2$ could rescue $L b x 1$ deficiency [8]. Although only limited information about downstream factors of $L b x$ is currently available [40], the comparative insights shown here suggest that skeletal muscles of vertebrate clades have deployed different combinations of $L b x 1$ and $L b x 2$ to ensure differentiation of complex musculature at variable timing of development.

\section{Conclusions}

Our results indicate that $L b x 1$-positive muscle precursors in the gnathostome ancestor would have been acquired to give rise to the novel, distally located skeletal 


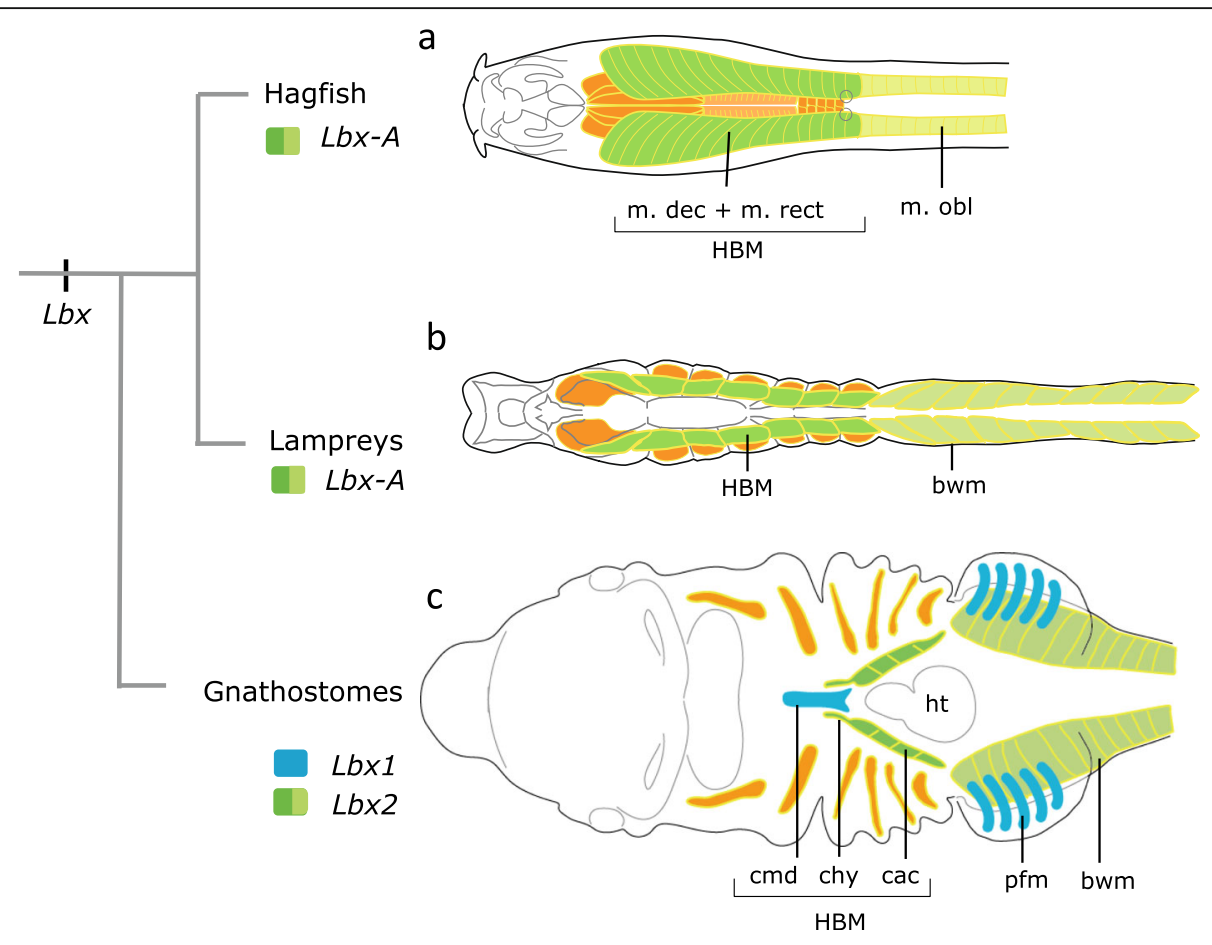

Fig. 5 Evolutionary scheme of vertebrate skeletal muscles with reference to the embryonic expression of Lbx genes. In the hagfish (a), rectus

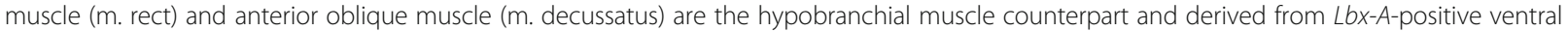
cells of the somites. In the lamprey (b), Lbx-A is required in the formation of body wall muscles (bwm, light green) and hypobranchial muscle (HBM, dark green). In gnathostomes (c), Lbx1 (blue) and Lbx2 (green) regulate distinct domains of hypobranchial muscles. Lbx1 functions in the tongue ( $\mathrm{cmd}$, $\mathrm{m}$. coracomandibularis in the shark) and appendicular muscles (pfm, pectoral fin muscles) both of which are lacking in cyclostomes. Pharyngeal muscles derived from head mesoderm are shown in orange

muscles, i.e., the muscles associated with paired appendages and the anterior internalized tongue (Fig. 5). On the other hand, the posterior portion of the HBM as well as the body wall muscles might represent hypaxial muscle having a deeper evolutionary origin that can be traced back to the common ancestor of vertebrates. Our results lead us to propose a new evolutionary hypothesis-namely, that HBMs, the developmental background of which has long been assumed to be similar to that of limb muscles, may have a closer evolutionary relationship with the abdominal muscle that extensively surrounds the trunk coelom, and that the morphological elaboration of HBMs would have been coupled with the functionalization of duplicated $L b x$ genes.

\section{Materials and methods}

\section{Obtaining embryos of lamprey, hagfish, and catshark}

Mature adults of the Japanese lamprey $L$. camtschaticum, the hagfish E. burgeri, and the cloudy catshark $S$. torazame were collected in Hokkaido, Shimane, and Ibaraki prefectures in Japan, respectively. Embryos of each animal were obtained as previously described [41]. Briefly, lamprey eggs were inseminated in vitro and reared at $9^{\circ}$, followed by embryonic staging according to Tahara [20]. The larvae were further cultured in mineral water as described in Higuchi et al. [41] until they reach $50 \mathrm{~mm}$ in body length. Hagfish embryos were collected from the sea floor and incubated in artificial sea water, followed by staging according to Dean [42]. Catshark embryos were laid in the fish tank and staged as described previously $[43,44]$. Embryos were fixed in $4 \%$ paraformaldehyde or Serra's fixative. The experiments were conducted according to the institutional and national guidelines for animal ethics, approved by the RIKEN Animal Experiments Committee.

\section{Identification of lamprey and catshark genes}

Although we had previously reported partial mRNA sequence of $L j L b x-A$ [10], we searched for the full-length sequence with reference to the genomic sequence [45]. A cDNA fragment covering the putative $\mathrm{N}$-terminal Met and the stop codon was obtained. We replaced the previously registered $L j L b x-A$ sequence with a new complete cDNA sequence under the same GenBank reference number (HM116241). The mRNA sequence of E. burgeri Lbx$A$ (LC506433) was predicted on the Ensemble Genome Database (transcript ID: ENSEBUT00000001721.1; http:// www.ensembl.org) and amplified from embryonic total RNA. We performed a search for genes of the catshark $S$. torazame (Lbx1, LC506430; Lbx2, LC506431; Sox9, 
LC506432) using the sequence archive Squalomix (https:// transcriptome.riken.jp/squalomix/) containing the products of whole genome sequencing [46]. The corresponding cDNAs were amplified from poly-A RNA of stage 24-30 embryos.

\section{Phylogenetic analysis}

Full-length amino acid sequences (summarized in Additional file 2: Table S1) were aligned and phylogenetic trees were constructed by the neighbor-joining method [47] using ClustalX software (http://www.clustal.org) [48]. Positions with gaps were excluded from the analysis. The length of the branches is proportional to the phylogenetic distances estimated using Kimura's empirical method for protein distances [49]. The scale bar indicates an evolutionary distance of 0.05 amino acid substitution per position in the sequence. The degree of support for internal branches of the tree was assessed in 1000 bootstrap replicates [50].

\section{Immunohistochemistry}

To visualize differentiated muscles and neurons, we used the primary antibodies MF20 or A4.1025 (DSHB, University of Iowa) and anti-acetylated tubulin (T6793; SigmaAldrich). ZO-1 (61-7300; Thermo Fisher Scientific) was used to detect tight junctions. The samples were subsequently reacted with the secondary antibody, the $F(a b$ ') fragment of goat anti-mouse IgG $(\mathrm{H}+\mathrm{L})$ conjugated with horseradish peroxidase (Thermo Fisher Scientific). Sections were then counterstained with either hematoxylin or DAPI.

\section{In situ hybridization}

Whole-mount and section in situ hybridization of lamprey, hagfish, and catshark embryos was performed as previously described [10, 41]. In brief, paraffin-waxembedded sections of embryos were deparaffinized, treated with proteinase $\mathrm{K}$, and hybridized with DIGlabeled RNA probe overnight. Hybridized probes were detected with anti-DIG-AP Fab fragments and visualized with reaction with BM purple AP substrate (11442074001; Roche). Sections were counterstained with Nuclear Fast Red (H-2403; Vector Laboratories). The probe sequence of $L j L b x-A$ is highlighted in Additional file 1: Fig. S2. The probe template for $E b L b x-A$ was amplified with the primers 5'-CGCCCTGGAGGAACTCGCGAG-3' and 5'CATTGCCCTTAGCGTAAAGCF-3'. For the catshark, to distinguish the expression of $L b x 1$ and $L b x 2$, probes were designed to include the regions highly diverged between the two genes. Primers used to amplify the probe templates were Lbx1, 5'-GGACCTGGAGGAGA TGAAGG-3' and 5'-GACATGCGATGCAACAAGG-3'; $L b x 2,5^{\prime}$-CGAAGGGACTATTCCTTCTCT-3' and 5'AGTCCCACCCCTTCCCCAACAA-3'.

\section{Knock-down experiment of lamprey $L j L b x-A$ gene}

Fertilized eggs of the lamprey were microinjected at the 1cell stage with a mixture of Cas9 protein $(0.1 \mathrm{ng} / \mathrm{nl}$, PNA Bio) and guide RNA (gRNA, $25 \mathrm{pg} / \mathrm{nl}$ ) dissolved in PBS. At appropriate stages, the posterior tail of each embryo was amputated and processed for genomic DNA extraction and PCR amplification for the $L j L b x-A$ locus (Additional file 1: Fig. S5). The anterior body part of each embryo was fixed in 4\% PFA or Serra's fixative. After the genomic analyses, individuals with a high deletion rate were examined for phenotypes. As experimental controls, uninjected embryos, embryos injected with Cas9 only, and injected embryos with a low deletion rate were also analyzed and compared (Additional file 1: Fig. S8).

\section{Library preparation for the screening of CRISPR/Cas9- induced mutants by next-generation sequencing}

Each embryo was analyzed for the presence or absence of mutations in the $L j L b x-A$ genomic locus by targeted PCR amplification followed by sequencing with Illumina MiSeq. We followed the previously described procedure [51] with modifications. To increase the number of reads and the quality of reads compared to the original proto$\mathrm{col}$, we removed the self-complementary sequence region from one end of the PCR products before the Illumina library preparation (Additional file 1: Fig. S5). The first PCR was performed with 22 cycles using $10 \mathrm{ng}$ of a template DNA extracted from each embryo, with a gene-specific primer containing a 16-nt-long head sequence (head-GS primers; 5'- GCTATGCGCGAGCT GCgtcgagatggactcgctatt-3' and 5' - GCTATGCGCG AGCTGCgcacgcacgccgggcgacat-3'; Additional file 1: Fig. S2), and the KAPA HiFi HotStart ReadyMix (KAPA Biosystems). Products of each reaction were purified using the AMPureXP beads (Beckman Coulter). The second PCR was performed with 18 cycles, using the full volume of DNA amplified in the first PCR, a head primer containing an 8-nt-long barcode sequence (barcode-head primer; Additional file 3: Table S2), and the KAPA HiFi HotStart ReadyMix. Products of each reaction were purified using AMPureXP beads, quantitated with the Qubit dsDNA HS Assay kit (ThermoFisher Scientific), and combined. Annealing of the GS primer was performed by adding a 200 -fold molar amount of the primer to the combined PCR products of $20 \mathrm{ng}$ in a reaction mixture of $24 \mu \mathrm{l}$ containing $1 \mathrm{x}$ ThermoPol Reaction Buffer (NEB), $6 \mathrm{mM} \mathrm{MgSO}_{4}$, and $1.4 \mathrm{mM}$ dNTP mix, followed by incubation at $95^{\circ} \mathrm{C}$ for $1 \mathrm{~min}, 60^{\circ} \mathrm{C}$ for $1 \mathrm{~min}$, and snap-cooling on ice. Primer extension was performed by adding $1 \mu \mathrm{l}(8 \mathrm{U})$ of the Bst DNA polymerase (NEB) to the reaction mixture, followed by incubation at $65^{\circ} \mathrm{C}$ for $3 \mathrm{~min}$ and $80^{\circ} \mathrm{C}$ for $20 \mathrm{~min}$, and snap-cooling on ice. The combined PCR product, after the primer extension, was purified using AMPureXP beads, eluted in $10 \mu \mathrm{l}$ of EB 
buffer (Qiagen), and further treated with $36 \mathrm{U}$ of S1 nuclease (Takara) in a reaction mixture of $20 \mu$ containing $1 x$ S1 nuclease buffer at $23^{\circ} \mathrm{C}$ for $15 \mathrm{~min}$. The reaction was terminated by adding $0.5 \mu \mathrm{l}$ of $0.5 \mathrm{M}$ EDTA solution, and the DNA was purified using the AMPureXP beads and eluted in $10 \mu \mathrm{l}$ of buffer EB. The combined PCR product after primer extension and digestion of the singlestranded region was analyzed by TapeStation (Agilent) to confirm the shortened length of the head and the barcode sequences $(24 \mathrm{nt})$ before Illumina library preparation. Library preparation was performed as described previously [52] using $10 \mathrm{ng}$ of the combined PCR product that does not contain the self-complementary region, together with the Illumina TruSeq compatible adapter.

\section{Amplicon sequencing and detection of deletion on target regions}

Sequencing was performed using the library prepared above, without the use of the control PhiX library, on Illumina MiSeq with the MiSeq Reagent Nano Kit v2 (Illumina) to obtain 250-nt-long paired-end reads. Quality control of the sequencing reads was performed using FastQC ver. 0.11 .5 (https://www.bioinformatics.babraham.ac.uk/projects/fastqc/). The BBmerge function of BBTools ver. 37.88 [53] was used to merge two overlapping paired reads into a single long read. The merged reads were then trimmed to remove low-quality regions and the Illumina adapter sequences using Trim galore ver. 0.4.2 (https://www.bioinformatics.babraham.ac.uk/ projects/trim_galore/). After separating the amplicon reads according to the barcode sequence for each individual (Additional file 3: Table S2), Cutadapt ver. 1.8.3 [54] was used to remove the barcode sequence and the head sequence. The trimmed reads were aligned with the cDNA sequence of LjLbx-A by BBMap of BBTools, and deletion profiles of the CRISPR/Cas9 target sites were visualized and evaluated with the UCSC Integrative Genomics Viewer (IGV) ver. 2.7.2 [55]. The sequencing data have been deposited to the DNA Data Bank of Japan (DDBJ) under the accession number DRA009475.

\section{Supplementary information}

Supplementary information accompanies this paper at https://doi.org/10. 1186/s12915-020-00851-y.

\footnotetext{
Additional file 1: Figures. S1-S10. Skeletal muscle formation during lamprey embryogenesis (Fig. S1). Genomic structure of $L j L b x-A$ gene and positions of CRISPR/Cas9 targets (Fig. S2). Phylogenetic analysis of the cyclostome and catshark Lbx genes (Fig. S3). Expression of $L j L b x-A$ in the dorsal median fin muscle primordia (Fig. S4). Workflow and additional data for lamprey genome editing experiments (Fig. S5-S8). Expression of catshark $L b \times 2$ in the extending HBM precursor cells (Fig. S9). ZO-1 staining of HBM primordium in the catshark embryo (Fig. S10).

Additional file 2: Table S1. Lbx genes compared in this study.

Additional file 3: Table S2. Barcode primers used in lamprey genome editing experiments.
}

\section{Acknowledgements}

We thank Drs. Shigehiro Kuraku, Wataru Takagi, and Fumiaki Sugaraha for their valuable input on the genomic and morphological analyses, and Yayoi Nakai and Shunya Kuroda for technical assistances. We also thank Chiharu Tanegashima of the Laboratory for Phyloinformatics, RIKEN BDR, for the library preparation and MiSeq operation.

\section{Authors' contributions}

R.K., S.H, M.K., and S.K. conceived the project, designed the experiments, and wrote the paper. R.K., S.H., M.T., and O.N. performed the experiments. All authors analyzed and discussed the data. All authors approved the final version of the manuscript.

\section{Funding}

This work has been funded by Grant-in-Aid for Scientific Research (C) (19K06683) and Takeda Science Foundation to R.K. and Grant-in-Aid for Scientific Research on Innovative Areas (Research in a Proposed Research Area) (17H06385) to S.K.

\section{Availability of data and materials}

Newly sequenced genes have been registered in GenBank under the reference numbers shown in the "Materials and methods" section. The amplicon sequencing data of CRISPR/Cas9 experiments have been deposited to the DNA Data Bank of Japan (DDBJ) under the accession number DRA009475 [56]. DNA sequences used in the phylogenetic analyses are listed in Additional file 2: Table S1.

\section{Ethics approval and consent to participate}

Not applicable.

\section{Competing interests}

The authors declare that they have no competing interests.

\section{Author details}

${ }^{1}$ Laboratory for Evolutionary Morphology, RIKEN Center for Biosystems Dynamics Research (BDR), 2-2-3 Minatojima-minami, Chuo-ku, Kobe, Hyogo 650-0047, Japan. ${ }^{2}$ Department of Biology, Graduate School of Science, Kobe University, Kobe 657-8501, Japan. ${ }^{3}$ Department of Molecular Biology and Biochemistry, Graduate School of Biomedical \& Health Sciences, Hiroshima University, Hiroshima 734-8553, Japan. ${ }^{4}$ Laboratory for Phyloinformatics, RIKE N Center for Biosystems Dynamics Research (BDR), Kobe 650-0047, Japan. ${ }^{5}$ Evolutionary Morphology Laboratory, RIKEN Cluster for Pioneering Research (CPR), Kobe 650-0047, Japan.

Received: 9 July 2020 Accepted: 18 August 2020

Published online: 09 September 2020

\section{References}

1. Mackenzie S, Walsh FS, Graham A. Migration of hypoglossal myoblast precursors. Dev Dyn. 1998;213(4):349-58.

2. Kuratani S. Spatial distribution of postotic crest cells defines the head/trunk interface of the vertebrate body: embryological interpretation of peripheral nerve morphology and evolution of the vertebrate head. Anat Embryol (Berl). 1997;195(1):1-13.

3. Christ B, Ordahl CP. Early stages of chick somite development. Anat Embryol (Berl). 1995;191(5):381-96.

4. Dietrich S, Schubert FR, Healy C, Sharpe PT, Lumsden A. Specification of the hypaxial musculature. Development. 1998;125(12):2235-49.

5. Brohmann $\mathrm{H}$, Jagla $\mathrm{K}$, Birchmeier $\mathrm{C}$. The role of $\mathrm{Lbx} 1$ in migration of muscle precursor cells. Development. 2000;127(2):437-45.

6. Gross MK, Moran-Rivard L, Velasquez T, Nakatsu MN, Jagla K, Goulding M. Lbx1 is required for muscle precursor migration along a lateral pathway into the limb. Development. 2000;127(2):413-24.

7. Chen F, Liu KC, Epstein JA. Lbx2, a novel murine homeobox gene related to the Drosophila ladybird genes is expressed in the developing urogenital system, eye and brain. Mech Dev. 1999;84(1-2):181-4.

8. Wotton KR, Schubert FR, Dietrich S. Hypaxial muscle: controversial classification and controversial data? Results Probl Cell Differ. 2015;56:25-48.

9. Wei K, Chen J, Akrami K, Sekhon R, Chen F. Generation of mice deficient for Lbx2, a gene expressed in the urogenital system, nervous system, and Pax3 dependent tissues. Genesis. 2007;45(6):361-8. 
10. Kusakabe R, Kuraku S, Kuratani S. Expression and interaction of musclerelated genes in the lamprey imply the evolutionary scenario for vertebrate skeletal muscle, in association with the acquisition of the neck and fins. Dev Biol. 2011;350(1):217-27.

11. Adachi N, Pascual-Anaya J, Hirai T, Higuchi S, Kuratani S. Development of hypobranchial muscles with special reference to the evolution of the vertebrate neck. Zoological letters. 2018;4:5.

12. Green SA, Uy BR, Bronner ME. Ancient evolutionary origin of vertebrate enteric neurons from trunk-derived neural crest. Nature. 2017;544(7648):88-91.

13. Parker HJ, Bronner ME, Krumlauf R. A Hox regulatory network of hindbrain segmentation is conserved to the base of vertebrates. Nature. 2014; 514(7523):490-3.

14. Kuratani S, Kuraku S, Murakami Y. Lamprey as an evo-devo model: lessons from comparative embryology and molecular phylogenetics. Genesis. 2002; 34(3):175-83.

15. Kusakabe R, Kuratani S. Evolutionary perspectives from development of mesodermal components in the lamprey. Dev Dyn. 2007;236(9):2410-20.

16. Peters A, Mackay B. The structure and innervation of the myotomes of the lamprey. J Anat. 1961;95:575-85.

17. Oisi Y, Fujimoto S, Ota KG, Kuratani S. On the peculiar morphology and development of the hypoglossal, glossopharyngeal and vagus nerves and hypobranchial muscles in the hagfish. Zoological Lett. 2015;1:6.

18. Kuratani S, Ueki T, Aizawa S, Hirano S. Peripheral development of cranial nerves in a cyclostome, Lampetra japonica: morphological distribution of nerve branches and the vertebrate body plan. J Comp Neurol. 1997;384(4): 483-500.

19. Higashiyama H, Hirasawa T, Oisi Y, Sugahara F, Hyodo S, Kanai Y, Kuratani S. On the vagal cardiac nerves, with special reference to the early evolution of the head-trunk interface. J Morphol. 2016;277(9):1146-58.

20. Tahara Y. Normal stages of development in the lamprey, Lampetra reissneri (Dybowski). Zool Sci. 1988:5:109-18.

21. Horigome N, Myojin M, Ueki T, Hirano S, Aizawa S, Kuratani S. Development of cephalic neural crest cells in embryos of Lampetra japonica, with special reference to the evolution of the jaw. Dev Biol. 1999;207(2):287-308.

22. Kusakabe R, Takechi M, Tochinai S, Kuratani S. Lamprey contractile protein genes mark different populations of skeletal muscles during development. J Exp Zoolog B Mol Dev Evol. 2004;302(2):121-33.

23. Kusakabe R, Kuratani S. Evolution and developmental patterning of the vertebrate skeletal muscles: perspectives from the lamprey. Dev Dyn. 2005;234(4):824-34.

24. Chen F, Collin GB, Liu KC, Beier DR, Eccles M, Nishina PM, Moshang T, Epstein JA. Characterization of the murine Lbx2 promoter, identification of the human homologue, and evaluation as a candidate for Alström syndrome. Genomics. 2001;74(2):219-27.

25. Letelier J, de la Calle-Mustienes E, Pieretti J, Naranjo S, Maeso I, Nakamura T, Pascual-Anaya J, Shubin NH, Schneider I, Martinez-Morales JR, et al. A conserved Shh cis-regulatory module highlights a common developmental origin of unpaired and paired fins. Nat Genet. 2018;50(4):504-9.

26. Jagla K, Dolle P, Mattei MG, Jagla T, Schuhbaur B, Dretzen G, Bellard F, Bellard M. Mouse Lbx1 and human LBX1 define a novel mammalian homeobox gene family related to the Drosophila lady bird genes. Mech Dev. 1995:53(3):345-56.

27. Okamoto E, Kusakabe R, Kuraku S, Hyodo S, Robert-Moreno A, Onimaru K, Sharpe J, Kuratani S, Tanaka M. Migratory appendicular muscles precursor cells in the common ancestor to all vertebrates. Nat Ecol Evol. 2017;1(11):1731-6.

28. Turner N, Mikalauskaite D, Barone K, Flaherty K, Senevirathne G, Adachi N, Shubin $\mathrm{NH}$, Nakamura T. The evolutionary origins and diversity of the neuromuscular system of paired appendages in batoids. Proc Biol Sci. 2019; 286(1914):20191571

29. Wotton KR, Weierud FK, Dietrich S, Lewis KE. Comparative genomics of Lbx loci reveals conservation of identical Lbx ohnologs in bony vertebrates. BMC Evol Biol. 2008;8:171.

30. Haines L, Currie PD. Morphogenesis and evolution of vertebrate appendicular muscle. J Anat. 2001;199(Pt 1-2):205-9.

31. Goodrich ES. Studies on the structure and development of vertebrates; 1930.

32. Bultje RS, Castaneda-Castellanos DR, Jan LY, Jan Y-N, Kriegstein AR, Shi S-H. Mammalian Par3 regulates progenitor cell asymmetric division via notch signaling in the developing neocortex. Neuron. 2009;63(2):189-202.

33. Mayeuf-Louchart A, Montarras D, Bodin C, Kume T, Vincent SD, Buckingham M. Endothelial cell specification in the somite is compromised in Pax3positive progenitors of Foxc1/2 conditional mutants, with loss of forelimb myogenesis. Development. 2016;143(5):872-9.
34. Ziermann JM, Freitas R, Diogo R. Muscle development in the shark Scyliorhinus canicula: implications for the evolution of the gnathostome head and paired appendage musculature. Front Zool. 2017;14:31.

35. Martin BL, Harland RM. A novel role for $\mid \mathrm{bx} 1$ in Xenopus hypaxial myogenesis. Development. 2006;133(2):195-208.

36. Sabo MC, Nath K, Elinson RP. Lbx1 expression and frog limb development. Dev Genes Evol. 2009;219(11-12):609-12.

37. Ochi $H$, Westerfield M. Lbx2 regulates formation of myofibrils. BMC Dev Biol. 2009;9:13.

38. Sagarin KA, Redgrave AC, Mosimann C, Burke AC, Devoto SH. Anterior trunk muscle shows mix of axial and appendicular developmental patterns. Dev Dyn. 2019;248(10):961-8

39. Watanabe S, Kondo S, Hayasaka M, Hanaoka K. Functional analysis of homeodomain-containing transcription factor Lbx 1 in satellite cells of mouse skeletal muscle. J Cell Sci. 2007;120(Pt 23):4178-87.

40. Guo L, Yamashita H, Kou I, Takimoto A, Meguro-Horike M, Horike S, Sakuma T, Miura S, Adachi T, Yamamoto T, et al. Functional investigation of a noncoding variant associated with adolescent idiopathic scoliosis in zebrafish: elevated expression of the ladybird homeobox gene causes body axis deformation. PLoS Genet. 2016;12(1):e1005802.

41. Higuchi S, Sugahara F, Pascual-Anaya J, Takagi W, Oisi Y, Kuratani S. Inner ear development in cyclostomes and evolution of the vertebrate semicircular canals. Nature. 2019;565(7739):347-50.

42. Dean B. On the embryology of Bdellostoma stouti. A general account of myxinoid development from the egg and segmentation to hatching. In: Festschrift zum 70ten Geburststag Carl von Kupffer. Gustav Fischer; 1899. p. 220-76.

43. Adachi N, Kuratani S. Development of head and trunk mesoderm in the dogfish, Scyliorhinus torazame: I. embryology and morphology of the head cavities and related structures. Evol Dev. 2012;14(3):234-56.

44. Ballard WW, Mellinger J, Lechenault $\mathrm{H}$. A series of normal stages for development of Scyliorhinus-Canicula, the lesser spotted dogfish (Chondrichthyes, Scyliorhinidae). J Exp Zool. 1993;267(3):318-36.

45. Mehta TK, Ravi V, Yamasaki S, Lee AP, Lian MM, Tay BH, Tohari S, Yanai S, Tay A, Brenner S, et al. Evidence for at least six Hox clusters in the Japanese lamprey (Lethenteron japonicum). Proc Natl Acad Sci U S A. 2013;110(40):16044-9.

46. Hara Y, Yamaguchi K, Onimaru K, Kadota M, Koyanagi M, Keeley SD, Tatsumi K, Tanaka K, Motone F, Kageyama Y, et al. Shark genomes provide insights into elasmobranch evolution and the origin of vertebrates. Nat Ecol Evol. 2018;2(11):1761-71.

47. Saitou N, Nei M. The neighbor-joining method: a new method for reconstructing phylogenetic trees. Mol Biol Evol. 1987;4(4):406-25.

48. Thompson JD, Higgins DG, Gibson TJ. CLUSTAL W: improving the sensitivity of progressive multiple sequence alignment through sequence weighting, position-specific gap penalties and weight matrix choice. Nucleic Acids Res. 1994;22(22):4673-80.

49. Kimura M. Molecular evolutionary rates contrasted with phenotypic evolutionary rates. In: Kimura M, editor. The neutral theory of molecular evolution. Cambridge: Cambridge University Press; 1983. p. 55-97.

50. Felsenstein J. Confidence limits on phylogenies: an approach using the bootstrap. Evolution. 1985;39(4):783-91.

51. Herbold CW, Pelikan C, Kuzyk O, Hausmann B, Angel R, Berry D, Loy A. A flexible and economical barcoding approach for highly multiplexed amplicon sequencing of diverse target genes. Front Microbiol. 2015;6:731.

52. Kadota M, Hara Y, Tanaka K, Takagi W, Tanegashima C, Nishimura O, Kuraku S. CTCF binding landscape in jawless fish with reference to Hox cluster evolution. Sci Rep. 2017;7(1):4957

53. Bushnell B, Rood J, Singer E. BBMerge - accurate paired shotgun read merging via overlap. PLoS One. 2017;12(10):e0185056.

54. Martin M. Cutadapt removes adapter sequences from high-throughput sequencing reads. EMBnet J. 2011;17:10-2. https://doi.org/10.14806/ej.17.1.200.

55. Thorvaldsdottir H, Robinson JT, Mesirov JP. Integrative Genomics Viewer (IGV): high-performance genomics data visualization and exploration. Brief Bioinform. 2013;14(2):178-92.

56. Kusakabe R, Higuchi S, Tanaka M, Kadota M, Nishimura O, Kuratani S. Novel developmental bases for the evolution of hypobranchial muscles in vertebrates. Supplementary Datasets. 2020. DDBJ accession DRA009475: https://ddbj.nig.ac.jp/DRASearch/submission?acc=DRA009475.

\section{Publisher's Note}

Springer Nature remains neutral with regard to jurisdictional claims in published maps and institutional affiliations. 